\title{
Reutivar: App para la programación óptima de fertirriego con agua regenerada
}

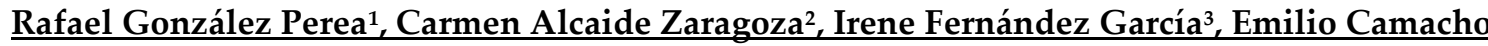 \\ Poyato $^{4}$, Juan Antonio Rodríguez Díaz1 \\ 1Departamento de Agronomía. Universidad de Córdoba. Campus Rabanales, Edif. Leonardo da Vinci, 14071. Córdoba. \\ g72goper@uco.es. \\ ${ }^{2}$ Departamento de Agronomía. Universidad de Córdoba. Campus Rabanales, Edif. Leonardo da Vinci, 14071. Córdoba. \\ g12alzac@uco.es. \\ ${ }^{3}$ Departamento de Ingeniería eléctrica, Universidad de Córdoba. Campus de Rabanales, Edif. Leonardo da Vinci. \\ g52fegai@uco.es. \\ ${ }^{4}$ Departamento de Agronomía. Universidad de Córdoba. Campus Rabanales, Edif. Leonardo da Vinci, 14071. Córdoba. \\ ecamacho@uco.es. \\ ${ }^{5}$ Departamento de Agronomía. Universidad de Córdoba. Campus Rabanales, Edif. Leonardo da Vinci, 14071. Córdoba. \\ ma2rodij@uco.es.
}

Resumen: El olivar es un cultivo de gran repercusión económica en toda España, siendo el país con la mayor superficie del mundo dedicada a éste. Esta importancia se acentúa en Andalucía, donde es el cultivo más representativo e icónico en la región. De hecho, debido a la superficie que ocupa, el olivar es el cultivo que más agua consume en toda la Cuenca Hidrográfica del Guadalquivir. En cuanto a su fertilización, ésta suele ser poco precisa y, con frecuencia, basada en prácticas culturales y en la experiencia del agricultor. Esto conlleva que se realicen aplicaciones de fertilizantes innecesarias, ocasionando la contaminación de suelos y aguas subterráneas. En este escenario, la utilización de agua regenerada para riego de olivar surge como alternativa a las fuentes de agua tradicionales. Sin embargo, uno de los problemas asociados al riego con este tipo de agua es la gestión de la fertilización, pues ésta ya incorpora una importante cantidad de nutrientes, que es además variable a lo largo del año. Por ello, en este trabajo, se ha desarrollado una aplicación móvil (Reutivar-App) para la programación conjunta y en tiempo real del riego y la fertilización del olivar considerando el riego con agua regenerada. Reutivar-App está destinada a técnicos y agricultores e integra metodologías complejas y ya consolidadas, pero en un formato muy amigable. La aplicación tiene en cuenta tanto registros agroclimáticos históricos como predicciones climáticas, características hidráulicas del sistema de riego, dotación de agua concedida, análisis foliares y análisis de calidad del agua periódicos. Como resultado, genera una recomendación semanal del tiempo de riego y la cantidad de fertilizante óptimos a aplicar. Dichos resultados ponen de manifiesto que se puede hacer un uso mucho más eficiente del agua y el fertilizante y que, gracias a los nutrientes que este tipo de aguas aporta, el uso de fertilizantes adicionales puede ser innecesario, implicando importantes beneficios tanto medioambientales como económicos.

Palabras clave: App; programación de fertirriego; reutilización aguas regeneradas; olivar. 


\section{INTRODUCCIÓN}

En España, alrededor del $70 \%$ del agua dulce total distribuida va destinada a la agricultura de regadío (FAO, 2012) lo cual hace a esta actividad especialmente vulnerable ante periodos de sequía. Según el World Resources Institute (2015), España experimentará un estrés hídrico severo para 2040, convirtiéndose así en uno de los países con mayor presión sobre los recursos hídricos a nivel mundial. Además, si en este contexto se tienen en cuenta también las previsiones futuras de aumento de la demanda de agua y del déficit potencial de humedad del suelo en diferentes zonas regables (Plan Hidrológico Guadalquivir, 2016), en los próximos años la agricultura de regadío tendrá que evolucionar para hacer frente a estos desafíos. Esta situación es especialmente crítica en zonas como Andalucía, donde la agricultura de regadío juega un papel fundamental. El cultivo más representativo y simbólico de Andalucía es el olivar, pues ha marcado su paisaje y su cultura y constituye, además, la base de la economía de muchas poblaciones. El olivar, debido a su extensión, y aun siguiendo estrategias de riego deficitario, es el cultivo con mayor demanda hídrica de la Demarcación Hidrográfica del Guadalquivir (más de $580 \mathrm{hm}^{3} /$ año), lo que supone algo más del $20 \%$ del total agrícola. Por otro lado, la fertilización de este cultivo se realiza generalmente de forma poco precisa, siguiendo prácticas culturales tradicionales y sin control sobre la humedad del suelo o del agua aplicada. Esto provoca aplicaciones de fertilizantes en exceso, especialmente de nitrógeno, lo que ocasiona problemas de contaminación del suelo y de las aguas. Este problema puede agravarse en el caso de riego con aguas residuales depuradas, pues estas ya incorporan una cantidad importante de nutrientes la cual, además, es variable a lo largo del año. Sin embargo, la utilización de este tipo de aguas, gestionadas correctamente y con ayuda de los nuevos avances en tecnología y comunicación, puede convertirse en una solución estratégica a la problemática planteada.

Los impactos asociados al uso de aguas regeneradas utilizadas para riego de olivar han sido considerados por varios autores, centrando su atención en los impactos en el suelo (Batarseh et al., 2011; Petousi et al., 2015; Segal et al., 2011), en el desarrollo del árbol (Ayoub et al., 2016; Bedbabis et al., 2010) y en la calidad del aceite (Palese et al., 2006; Bedbabis et al., 2010; Bourazanis et al., 2016), concluyendo que el uso de estas aguas, siempre que se haga de manera controlada y se realicen los pertinentes análisis regularmente, no afecta a ninguno de los parámetros evaluados.

Por otro lado, diferentes autores han desarrollado aplicaciones enfocadas a la gestión del uso del agua y la fertilización. Bueno-Delgado et al. (2016) y Pagán et al. (2015) propusieron una metodología para determinar el tipo de fertilizante que debería emplearse desde el punto de vista económico, teniendo en cuenta la calidad del agua de riego. Sin embargo, en ambos trabajos es necesario conocer la solución nutritiva ideal como parámetro de entrada, la cual el agricultor suele desconocer. Pérez-Castro et al. (2017) desarrollaron un método para determinar la cantidad de fertilizantes que se debe aplicar según el tipo de cultivo y las condiciones del sistema, pero solo es aplicable a cultivos hortícolas en invernadero. En cuanto a la mejora de la gestión del uso del agua, González Perea et al. (2017) implementaron los principios de riego de precisión para el cultivo de fresa en una aplicación de fácil manejo para el agricultor, lo que conllevó importantes ahorros de agua. Mérida García et al., (2018) presentaron un modelo de gestión óptima del riego de olivar mediante energía solar que ajustaba el riego según las necesidades de riego del cultivo en tiempo real. Sin embargo, no existe ninguna herramienta de gestión, de fácil manejo, que integre todos los elementos anteriormente citados: gestión del uso del agua y el fertilizante, es decir, un fertirriego de precisión para el cultivo de olivar usando aguas regeneradas. 


\section{OBJETIVOS}

El objetivo de este trabajo es el desarrollo de una aplicación de escritorio y para dispositivos móviles, destinada a los agricultores, que realice la programación en tiempo real del fertirriego de precisión del olivar regado con aguas regeneradas mediante en el uso de las Tecnologías de la Información y la Comunicación (TICs), maximizando la eficiencia en el uso del agua y el fertilizante y asegurando la sostenibilidad del olivar de regadío.

\section{MATERIALES Y MÉTODOS}

\subsection{Caso de estudio}

El estudio se ha llevado a cabo en el Sector I de la Comunidad de Regantes (C.R.) de Tintín, localizado en Montilla (Córdoba), concretamente en una finca comercial de olivar tradicional. En esta zona, el clima es mediterráneo, con una precipitación media anual $\left(P_{t}\right)$ de $590 \mathrm{~mm}$, principalmente en primavera y otoño. La temperatura media anual $\left(T_{m}\right)$ es de $16.9^{\circ} \mathrm{C}$ y la evapotranspiración de referencia media $\left(E T_{0}\right)$ es $3 \mathrm{~mm}$ día ${ }^{-1}$. La finca está compuesta por árboles adultos (Olea europea L., cv Hojiblanca y Olea europea L., cv Nevadillo azul) espaciados a $8 \mathrm{~m} \times 8 \mathrm{~m}$ para la producción de aceite. El agua de riego, procedente de la EDAR de Montilla, se almacena en una balsa y desde allí se distribuye a los regantes mediante una estación de bombeo localizada aguas abajo de dicha balsa. El sistema de riego empleado es riego por goteo subterráneo, con goteros de $2.2 \mathrm{~L} / \mathrm{h}$ y espaciados $1 \mathrm{~m}$.

\subsection{Caracterización de la finca}

La caracterización de la finca contempló la caracterización hidráulica del sistema de riego, del suelo, del estado nutricional de los árboles y de la calidad del agua de riego. La caracterización hidráulica parte del levantamiento de la finca y su posterior digitalización. A continuación, se realizó la simulación hidráulica del sistema de riego en EPANET (Rossman, 2000) para obtener la eficiencia de riego de la parcela. Respecto a la caracterización del suelo, se tomaron ocho muestras de suelo en cuatro puntos diferentes, considerando cambios en la morfología, color y pendiente. Dos de esos puntos se localizaron bajo el árbol y los dos restantes correspondieron a muestras de suelo procedente de las calles. En cada punto seleccionado se tomaron dos muestras, una en la capa de suelo de $0-15 \mathrm{~cm}$ y la otra en la de $15-30 \mathrm{~cm}$ para evaluar textura, $\mathrm{pH}$ y contenido de nutrientes. Para la caracterización del estado nutricional del árbol se realizaron análisis foliares en el mes de julio. Se tomaron dos muestras, correspondientes a las dos variedades de olivo presente en la finca, formadas por 100 hojas de 50 árboles cada una. Finalmente, para la evaluación de la calidad de agua de riego se tomaron muestras en parcela del agua aplicada cada dos semanas y se analizaron posteriormente en el laboratorio.

\subsection{Descripción del modelo}

El objetivo del modelo es proporcionar en tiempo real a los agricultores, la cantidad de agua y fertilizante necesaria a aportar, ajustando estos valores a las necesidades reales del cultivo para mejorar la sostenibilidad del sistema productivo. El modelo, denominado REUTIVAR, se ha desarrollado en Android OS y está formado por cinco módulos independientes e interconectados: 1) características de la plantación, 2) datos climáticos, 3) limitación mensual de uso de agua, 4) programación del riego y 5) programación de la fertilización (Figura 1). Los módulos que conforman el modelo se describen a continuación.

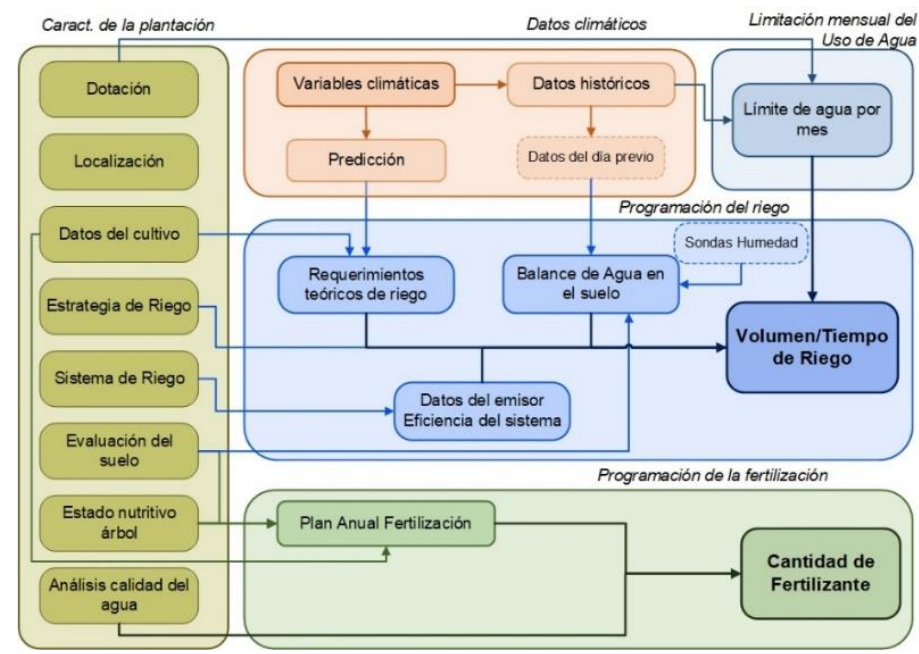

Figura 1. Esquema general del modelo REUTIVAR 


\subsubsection{Características de la plantación}

En este módulo se introducen los datos generales de la plantación tales como localización de la parcela, características texturales del suelo, ciclo del cultivo, coeficiente de cultivo, dotación de riego concedida, características del sistema de riego (caudal, separación entre goteros, etc.), estrategia de riego, días de la semana que se quiere regar, análisis del estado nutritivo del árbol (mediante análisis foliares) y análisis de la calidad del agua de riego.

\subsubsection{Datos climáticos}

Este módulo contempla dos aspectos: predicción meteorológica y registros agroclimáticos históricos, los cuales dependen de la localización de la parcela de cultivo. En primer lugar, se obtiene, de forma diaria, la predicción meteorológica de la zona de estudio para una semana utilizando AEMET OpenData, la API REST (Application Programming Interface. REpresentational State Transfer) que pone a disposición la Agencia Estatal de Meteorología (AEMET, 2015). Los datos que se obtienen son: precipitación $(P)$, temperatura media $\left(T_{m}\right)$, temperatura máxima $\left(T_{m a x}\right)$, temperatura mínima $\left(T_{\min }\right)$, humedad relativa máxima $\left(H R_{\max }\right)$, humedad relativa mínima $\left(H R_{\min }\right)$, velocidad del viento $\left(u_{2}\right)$ e índice de nubosidad $(n u)$. A partir de estos parámetros y mediante la ecuación de FAO Penman-Monteith (Allen, 1998), se obtiene el valor de la evapotranspiración de referencia, $\mathrm{ET}_{0}$.

Así mismo, se determina la estación agroclimática más cercana a la localización seleccionada de las disponibles en la Red de Estaciones Agroclimáticas de Andalucía. De esta estación, mediante técnicas de web scraping, se obtienen los valores diarios de $\mathrm{P}$ y ETo de todos los años disponibles de la estación $y$, diariamente, se almacenan estos mismos datos del día anterior. Toda esta información se incorpora en una base de datos integrada en la aplicación, la cual se usa para la programación de los eventos de riego.

\subsubsection{Disponibilidad mensual de agua}

En este módulo, se establecen límites de la cantidad de agua que se puede aplicar en cada periodo. Estos límites dependen de la concesión de uso de aguas regeneradas, de la estrategia de riego que se adopte y de las necesidades de riego del cultivo a lo largo de su ciclo productivo, calculadas a partir de las características del cultivo y de la serie histórica de datos climáticos. El límite de agua aplicable es variable a lo largo de los meses. De forma quincenal, se comprueba que la cantidad de agua aportada no supera dicho límite. En caso de haber utilizado menos agua de la establecida inicialmente, el agua no usada se podrá aplicar en los meses venideros.

\subsubsection{Programación del riego}

La programación del riego se realiza considerando desde los requerimientos teóricos de riego del cultivo, la estrategia de riego seleccionada, el sistema de riego, el balance diario de agua en el suelo hasta la limitación mensual de agua a aplicar. Las necesidades teóricas de riego se calculan al final del día previo al día de riego, a partir de los datos de las predicciones meteorológicas, como la diferencia entre la evapotranspiración del cultivo $\left(E T_{c}\right)$ y la precipitación efectiva $\left(P_{\text {eff }}\right)$ del día de riego, que es el agua de lluvia almacenada en la zona radicular y aprovechable por el cultivo, se calcula por el método del Servicio de Conservación de Recursos Naturales del USDA. Las necesidades de riego del cultivo se calculan para reponer la $E T_{c}$ diaria, calculada por el método propuesto por la FAO (Doorenbos \& Pruit, 1977):

$$
E T_{c}=E T_{0} \cdot k_{c} \cdot k_{r}
$$

Donde $E T_{0}$, se calcula con la previsión meteorológica (ver apartado 3.3.2); $k_{c}$ es el coeficiente de cultivo, cuyos valores son los propuestos por Orgaz y Fereres (2001) para olivar (Tabla 1) y $k_{r}$ es un coeficiente de cubierta que varía de 0 a 1 para árboles con menos del $60 \%$ de cubierta y 1 cuando superan ese valor. En el caso de la finca de estudio, al tratarse de árboles adultos, este valor se considera 1.

Tabla 1. Coeficiente de cultivo $\left(k_{c}\right)$ del olivo

\begin{tabular}{cccccccccccc} 
Ene & Feb & Mar & Abr & May & Jun & Jul & Ago & Sept & Oct & Nov & Dic \\
\hline 0.65 & 0.65 & 0.65 & 0.60 & 0.55 & 0.55 & 0.50 & 0.50 & 0.55 & 0.55 & 0.60 & 0.65 \\
\hline
\end{tabular}


En cuanto a la estrategia de riego, el modelo ofrece tres posibles opciones al agricultor: riego completo $(\mathrm{RC})$, riego deficitario controlado (RDC) y riego deficitario sostenido (RDS). El RC considera el total de las necesidades del olivo. La estrategia de RDS distribuye equitativamente a lo largo de la campaña de riego un porcentaje de las necesidades totales del árbol. Finalmente, la estrategia de RDC también aplica un porcentaje de las necesidades de riego totales, pero con la diferencia de que estos porcentajes son variables a lo largo de la campaña, concentrado la mayor cantidad de agua en las fases fenológicas más críticas del árbol, con el objetivo de minimizar los impactos en el crecimiento del árbol, rendimiento y calidad del aceite. Estos periodos se seleccionaron siguiendo los criterios establecidos por Rallo y Cuevas (2017) y Orgaz, Fereres y Testi, (2017). Para el caso particular de Andalucía, considerando los problemas asociados a las escasez de agua y a la importancia del olivar, la estrategia recomendada es la RDC (Moriana et al., 2003; Padilla-Díaz, et al. 2016).

Para la programación del riego, se hace un seguimiento de la humedad del suelo. Para ello, se calcula el Balance de Agua en el Suelo (BAS) de forma diaria (Ec. (2)). En el caso de disponer de sondas de humedad, como sucede en la finca de estudio, el balance de agua en el suelo calculado se retroalimenta semanalmente con los datos de las sondas de humedad instaladas en campo.

$$
B A S_{d}=B A S_{d-1}+P_{e f f}+I_{a p}-E T_{c, a d j}-R-D
$$

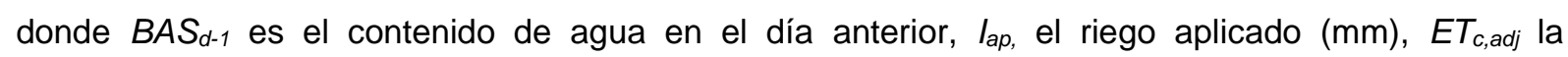
evapotranspiración del cultivo ajustada $(\mathrm{mm}), R$ la escorrentía $(\mathrm{mm})$ y $D$ la percolación profunda $(\mathrm{mm})$. $R$ y $D$ son considerados nulos en este caso, ya que se trata de riego por goteo.

La $E T_{c, a d j}$ está relacionada con la dificultad del cultivo para extraer agua cuando la humedad del suelo no es alta. En el modelo se considera este efecto mediante la Ec. 3 (Allen, 1998):

$$
E T_{c, a d j}=E T_{c} \cdot \frac{A T D-D_{r}}{A T D-A F A}
$$

donde $A T D$ es el agua total disponible del suelo $(\mathrm{mm}), D_{r}$, el agotamiento en la zona radicular $(\mathrm{mm})$, calculado como la diferencia entre $A T D$ y $B A S_{d-1}$, y $A F A$ es el agua fácilmente aprovechable en el suelo $(\mathrm{mm})$.

Por último, se calcula el tiempo de riego $t$ (h) según la Ec.(4):

$$
t=\frac{H_{r} \cdot A_{s} \cdot 10^{4}}{I E \cdot \sum_{i=1}^{e} q_{e} \cdot n_{e}}
$$

donde $H_{r}$ son los requerimientos de riego (mm), $A_{s}$ es el área del sector (ha), IE la eficiencia de riego, $q_{e}$ el caudal del gotero (L/h) y $n_{\mathrm{e}}$ el número de goteros del sector.

\subsubsection{Programación de la fertilización}

La planificación anual de la fertilización se basa en la evaluación del estado nutritivo del árbol, del contenido de nutrientes en suelo y de los nutrientes disueltos en el agua de riego (Fernández-Escobar, 2017). Está demostrado que las necesidades de fertilización del olivar son muy pequeñas y, en numerosas ocasiones, los nutrientes se encuentran en cantidades suficientes en el suelo para satisfacer sus necesidades. Si, además, se tiene en cuenta que los casos que aborda este trabajo son árboles regados con aguas residuales tratadas, es posible que se estén aportando algunos nutrientes a través del riego. Sin embargo, pueden darse situaciones en las que el suelo sea pobre en algún nutriente o que éstos estén bloqueados, por lo que el árbol no los puede asimilar. Por ello, es esencial la realización del análisis foliar del árbol para así determinar si hay exceso o carencia de nutrientes y, a partir de ahí, corregir. Es por esto por lo que, en primer lugar, es necesario conocer los valores de los niveles de nutrientes en hojas (obtenidos mediante los análisis foliares realizados el año anterior en el mes de julio) y sólo en el caso de ser deficiente (ver

Tabla 2) alguno de estos niveles, se aplica dicho elemento (Fernández-Escobar, 2018). Los elementos que se analizan en este trabajo son nitrógeno $(N)$, fósforo $(P)$ y Potasio $(K)$.

Tabla 2. Interpretación de los niveles de nutrientes en hojas de olivo recogidas en julio, expresados en \% de materia seca

\begin{tabular}{lccc} 
Elemento & Deficiente & Adecuado & Tóxico \\
\hline Nitrógeno (\%) & 1,2 & $1,3-1,7$ & $>1,7$ \\
Fósforo $(\%)$ & 0,05 & $0,1-0,3$ & -
\end{tabular}




\section{Congreso Nacional de Riegos CARTAGENA 2021}

En caso de deficiencia, la estrategia seguida se basa en la aplicación de una cantidad inicial, calculada a partir de una estimación del consumo del árbol de dicho elemento, en formato líquido (FernándezEscobar, 2017). Al año siguiente se vuelve a realizar el análisis foliar y se comprueba si es necesario aumentar o disminuir dicha dosis. En el caso del $\mathrm{N}$, si su nivel en hoja es menor de 1,3\% se aplican 0,5 $\mathrm{kg} /$ árbol de N, sin superar los $150 \mathrm{~kg} / \mathrm{ha}$. En el caso del K, cuando el valor sea inferior o igual a $0,7 \%$ en hoja, se aplicará $1 \mathrm{~kg} / a ́ r b o l$. Por último, en el caso del $\mathrm{P}$, el valor inicial de aplicación será de 0,5 $\mathrm{kg} / a ́ r b o l$. La aplicación se hace en primavera ya que su eficacia es mayor y además evita que queden restos en los goteros a final de campaña. Se recomienda que la aplicación sea semanal ya que está demostrado que su eficacia es igual que una aplicación diaria, pero se necesita menos mano de obra, aunque el usuario puede modificar esta opción y hacer coincidir la aplicación del fertilizante con la programación riego.

El siguiente aspecto considerado en el modelo es sobre la calidad de agua y los nutrientes que ya lleva en solución. En el caso de usar aguas regeneradas, el contenido en macronutrientes es considerable y debe restarse en el cálculo del fertilizante total a aplicar.

\subsection{Aplicación informática}

El modelo propuesto contempla el desarrollo de una aplicación para dispositivos móviles que implementa los módulos descritos previamente. Por otro lado, se ha desarrollado la versión beta de la aplicación en Android, la cual integra este mismo modelo y permite al usuario gestionar el riego de forma cómoda desde su smartphone. Los agricultores de la Comunidad de Regantes Tintín (Montilla) probarán la versión beta de aplicación durante la campaña de riego de 2019.
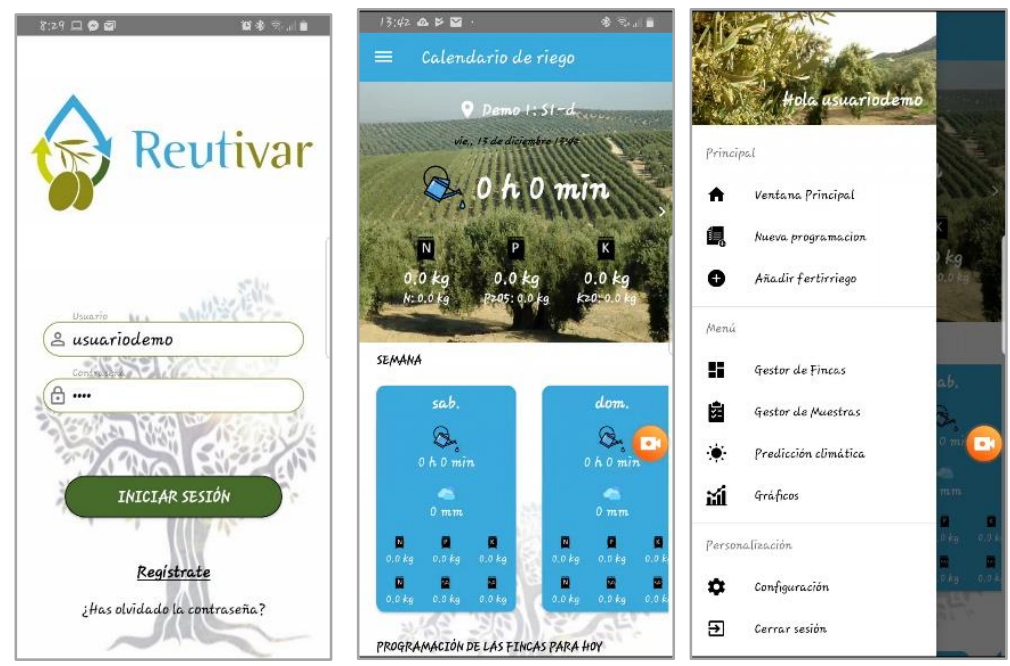

Figura 2. Interfaz gráfica de Reutivar

\section{RESULTADOS Y DISCUSIÓN}

A continuación, se muestran los resultados obtenidos al aplicar REUTIVAR a la campaña de riego 2018.

\subsection{Programación del riego}

La programación del riego para la campaña de riego 2018 se muestra en la

Figura 3. 


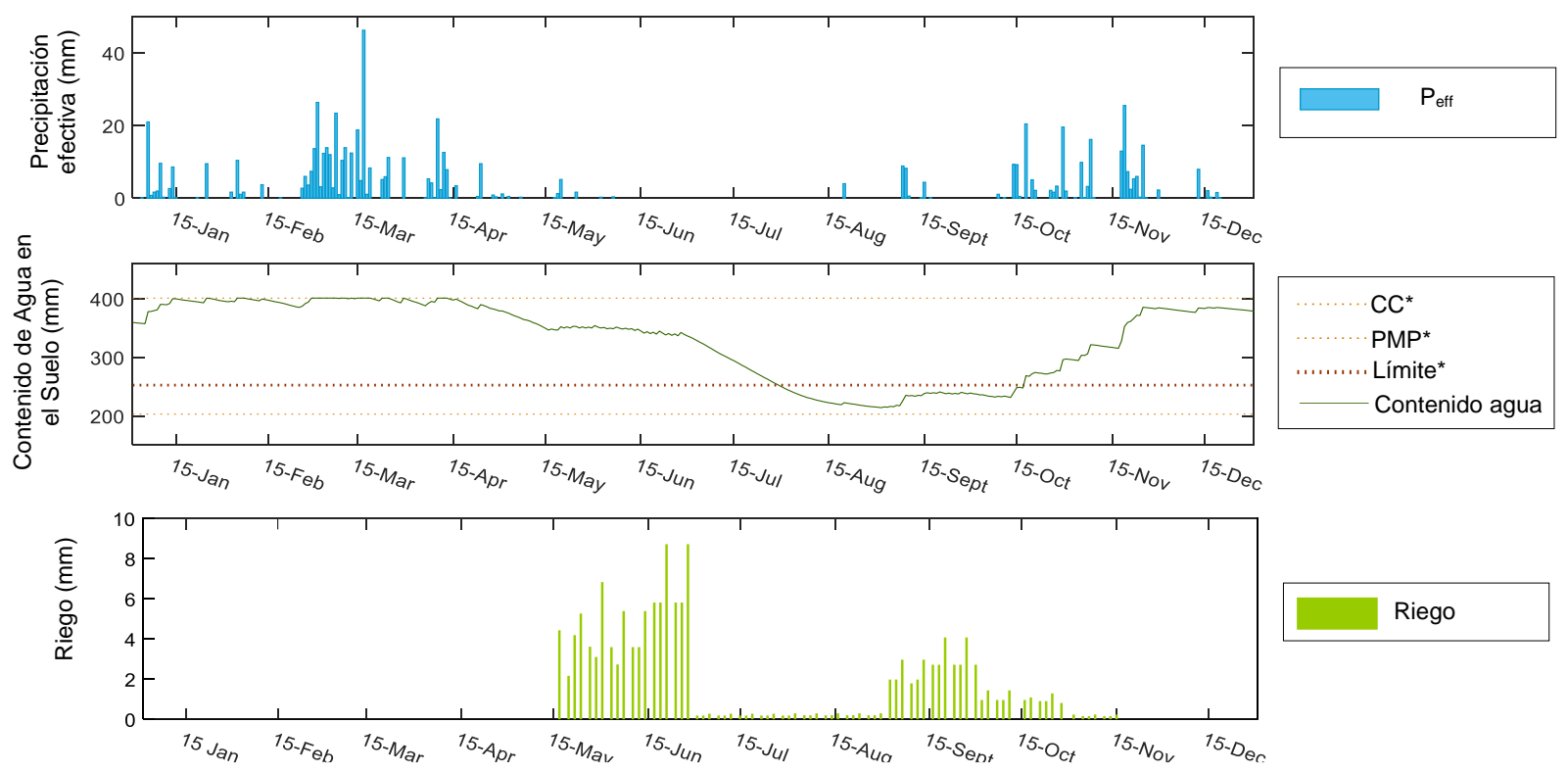

Figura 3. Distribución anual de la programación de riego propuesta, precipitación efectiva y contenido de agua en el suelo

* CC: Capacidad de Campo; PMP: Punto de Marchitez Permanente; Límite: 75\% de ATD, cuando deja de ser AFA. El modelo ofrece una programación del riego que distribuye la dotación concedida aplicando un total de $1.490 \mathrm{~m}^{3} /$ ha. Los momentos en los que se concentra el riego son mayo, junio y septiembre, momentos especialmente críticos al estrés hídrico (Rallo \& Cuevas, 2017), concentrándose el déficit en los meses de verano. En agosto y hasta mediados de octubre, el contenido de agua en el suelo es inferior al contenido de agua fácilmente aprovechable, por lo que el cultivo tendrá mayor dificultad para extraer el agua del suelo.

\subsection{Programación de la fertilización}

Los resultados relativos al análisis de suelo se observan en la Tabla 3.

Tabla 3. Resultados análisis de los análisis de suelo realizados en la finca de estudio

\begin{tabular}{crccccccc}
$\begin{array}{c}\text { Nombre } \\
\text { muestra }\end{array}$ & \multicolumn{1}{c}{$\begin{array}{c}\text { Espesor } \\
(\mathrm{cm})\end{array}$} & $\mathrm{pH}$ & $\begin{array}{c}\text { C.E. }(1: 5) \\
(\mathrm{dS} / \mathrm{m})\end{array}$ & Arcilla & Limo & Arena & $P(\mathrm{mg} / \mathrm{Kg})$ & $K(\mathrm{mg} / \mathrm{Kg})$ \\
\hline A1P1 & $0-15$ & 8.12 & 0.09 & 37.8 & 26.7 & 35.3 & 14.1 & 563 \\
A1P2 & $15-30$ & 8.31 & 0.10 & 38.1 & 28.0 & 33.9 & 7.7 & 454 \\
A2P1 & $0-15$ & 8.33 & 0.11 & 33.8 & 23.3 & 42.0 & 9.9 & 454 \\
A2P2 & $15-30$ & 8.20 & 0.10 & 37.4 & 20.0 & 42.7 & 5.8 & 317 \\
C1P1 & $0-15$ & 8.10 & 0.27 & 37.0 & 28.0 & 34.4 & 23.6 & 872 \\
C1P2 & $15-30$ & 8.10 & 0.48 & 34.2 & 28.6 & 36.1 & 25.7 & 794 \\
C2P1 & $0-15$ & 8.12 & 0.47 & 32.0 & 23.4 & 43.5 & 22.8 & 978 \\
C2P2 & $15-30$ & 8.00 & 0.59 & 36.1 & 21.2 & 42.1 & 27.0 & 598
\end{tabular}

De estos resultados se interpreta, en primer lugar, dado que el pH se encuentra comprendido en todas las muestras en un intervalo entre 8 y 8.3 , se considera que es un suelo alcalino. En estos valores, el olivo por lo general vegeta bien (Parra, 2017). En cuanto a la conductividad eléctrica (C.E.), que proporciona información sobre el contenido de sales, se observa un contenido mayor en las muestras procedentes de las calles, siendo este ligeramente salino. Sin embargo, se debe considerar que la toma de muestras se realizó en septiembre, antes de la época de lluvias, que lavarían el suelo posteriormente, reduciendo el contenido en sales, por lo que se considera que no hay problemas de salinidad. Del análisis textural del suelo mediante el triángulo textural del USDA, se obtuvo que el suelo en todas las muestras se correspondía con el tipo franco-arcilloso.

En cuanto al análisis de macronutrientes, la cantidad de $\mathrm{P}$ en las calles es alta mientras que en los árboles es menor, pero aun así se encuentra en un rango en el que es poco probable que haya 
respuesta al abonado, especialmente para el caso del olivo, pues la importancia de este nutriente es inferior a la de otros cultivos anuales, debido a sus bajas extracciones y su facilidad de reutilización. Además, se ha demostrado que el contenido en exceso de $\mathrm{P}$ en el suelo puede inducir bloqueos de Cinc. Finalmente, en cuanto al contenido de $\mathrm{K}$, los valores en todas las muestras son altos, por lo que, del mismo modo que en el caso del P, no se espera respuesta al abonado.

Los resultados de los análisis foliares llevados a cabo en el mes de julio de 2018 para dos variedades de olivo presentes en la finca de estudio se muestran en la Tabla 4.

Tabla 4. Resultados de los análisis foliares realizados en el mes de julio en la finca de estudio,

\begin{tabular}{lccc}
\multicolumn{1}{c}{ Variedad } & Nitrógeno $(\mathrm{g} / \mathrm{100g})$ & Fósforo $(\mathrm{g} / 100 \mathrm{~g})$ & Potasio $(\mathrm{g} / \mathrm{100g})$ \\
\hline Hojiblanca & 2,02 & 0,11 & 0,92 \\
Nevadillo azul & 1,35 & 0,13 & 0,88
\end{tabular}

Como se puede observar, en el caso de la variedad Nevadillo Azul, todos los valores se encuentran en los niveles adecuados. Por lo tanto, siguiendo los criterios comentados anteriormente y los resultados de los análisis de suelo, para la próxima campaña no se debería aplicar ningún fertilizante. En el caso de la variedad Hojiblanca, sin embargo, se observan valores adecuados de $\mathrm{P}$ y $\mathrm{K}$ en hoja, pero se observa que el valor de $\mathrm{N}$ es superior al nivel establecido. Numerosos estudios avalan la problemática ocasionada por un exceso de fertilización nitrogenada en olivar, tales como disminución de la calidad del aceite (Erel et al., 2013; Fernández-Escobar et al., 2006), mayor sensibilidad a las heladas (Fernández-Escobar, Navarro, \& Melgar, 2011) o retraso en la maduración del fruto, lo que suele relacionarse con una disminución del rendimiento graso (Fernández-Escobar, Antonaya-Baena, Sánchez-Zamora, \& Molina-Soria, 2014) . Por tanto, es aconsejable que para la campaña de 2019 no se aplique ningún abono.

\section{CONCLUSIONES}

El riego de olivar con aguas regeneradas es una alternativa al uso de fuentes de agua convencionales. Sin embargo, es esencial una correcta gestión de éstas. Para ello, se ha desarrollado una aplicación para dispositivos móviles de fácil manejo para la implantación de fertirriego de precisión, que optimiza el uso de aguas regeneradas, proporcionando de forma diaria la cantidad de agua y fertilizante que es necesario aportar. De este modo, se demuestra que el uso de este tipo de aguas para riego permite el ahorro de fertilizantes, lo que proporciona beneficios significativos tanto económicos como medioambientales.

\section{AGRADECIMIENTOS}

Este trabajo forma parte del proyecto REUTIVAR, proyecto de innovación cofinanciado por la Junta de Andalucía y la Unión Europea a través del FEADER 2014-2020, en el marco de las ayudas de la Línea Olivar para el funcionamiento de grupos operativos de la Asociación Europea de Innovación (AEI) en materia de productividad y sostenibilidad agrícola.

\section{BIBLIOGRAFÍA}

Agencia Estatal de Meteorología (AEMET). (2015). AEMET OpenData. Retrieved 14 February 2019, from http://www.aemet.es/es/datos_abiertos/AEMET_OpenData

Allen, R. G. (1998). FAO Irrigation and Drainage Paper Crop by. Irrigation and Drainage, 300(56), 300. https://doi.org/10.1016/j.eja.2010.12.001

Ayoub, S., Al-Shdiefat, S., Rawashdeh, H., \& Bashabsheh, I. (2016). Utilization of reclaimed wastewater for olive irrigation: Effect on soil properties, tree growth, yield and oil content. Agricultural Water Management, 176, 163-169. https://doi.org/10.1016/j.agwat.2016.05.035

Batarseh, M. I., Rawajfeh, A., loannis, K. K., \& Prodromos, K. H. (2011). Treated municipal wastewater irrigation impact on olive trees (Olea Europaea L.) at Al-Tafilah, Jordan. Water, Air, and Soil Pollution, 217(1-4), 185-196. https://doi.org/10.1007/s11270-010-0578-7

Bedbabis, S., Ferrara, G., Ben Rouina, B., \& Boukhris, M. (2010). Effects of irrigation with treated wastewater on olive tree growth, yield and leaf mineral elements at short term. Scientia Horticulturae, 126(3), 345-350. https://doi.org/10.1016/j.scienta.2010.07.020

Bedbabis, S., Rouina, B. Ben, \& Boukhris, M. (2010). The effect of waste water irrigation on the extra virgin olive oil quality from the Tunisian cultivar Chemlali. Scientia Horticulturae, 125(4), 556-561. https://doi.org/10.1016/j.scienta.2010.04.032

Bourazanis, G., Roussos, P. A., Argyrokastritis, I., Kosmas, C., \& Kerkides, P. (2016). Evaluation of the use of treated municipal 
waste water on the yield, oil quality, free fatty acids' profile and nutrient levels in olive trees cv Koroneiki, in Greece. Agricultural Water Management, 163, 1-8. https://doi.org/10.1016/j.agwat.2015.08.023

Bueno-Delgado, M. V., Molina-Martínez, J. M., Correoso-Campillo, R., \& Pavón-Mariño, P. (2016). Ecofert: An Android application for the optimization of fertilizer cost in fertigation. Computers and Electronics in Agriculture, 121, 32-42. https://doi.org/10.1016/j.compag.2015.11.006

CHG - Confederación Hidrográfica del Guadalquivir (Spanish Government). (2016). Plan Hidrológico de la demarcación hidrográfica del Guadalquivir. Ciclo de planificación hidrológica 2015-2021, 2015-2021.

Doorenbos, J., \& Pruit, W. O. (1977). Guidelines for predicting crop water requirements. FAO Irrigation and Drainage, 24.

Erel, R., Kerem, Z., Ben-Gal, A., Dag, A., Schwartz, A., Zipori, I., ... Yermiyahu, U. (2013). Olive (Olea europaea L.) tree nitrogen status is a key factor for olive oil quality. Journal of Agricultural and Food Chemistry, 61(47), 11261-11272. https://doi.org/10.1021/j44031585

FAO. (2012). AQUASAT database. Retrieved 12 September 2018, from http://www.fao.org/nr/water/aquastat/data/query/results.html

Fernández-Escobar, R. (2017). Fertilización. In El Cultivo del Olivo (pp. 419-460).

Fernández-Escobar, R. (2018). Trends in olive nutrition. Acta Horticulturae, 1199, $215-223$. https://doi.org/10.17660/ActaHortic.2018.1199.35

Fernández-Escobar, R., Antonaya-Baena, M. F., Sánchez-Zamora, M. A., \& Molina-Soria, C. (2014). The amount of nitrogen applied and nutritional status of olive plants affect nitrogen uptake efficiency. Scientia Horticulturae, 167, 1-4. https://doi.org/10.1016/j.scienta.2013.12.026

Fernández-Escobar, R., Beltrán, G., Sánchez-Zamora, M. A., García-Novelo, J., Aguilera, M. P., \& Uceda, M. (2006). Olive oil quality decreases with nitrogen over-fertilization. HortScience, 41(1), 215-219.

Fernández-Escobar, R., Navarro, S., \& Melgar, J. C. (2011). Effect of Nitrogen Status on Frost Tolerance of Olive Trees. Acta Horticulturae, (924), 41-45. https://doi.org/10.17660/ActaHortic.2011.924.3

González Perea, R., Fernández García, I., Martin Arroyo, M., Rodríguez Díaz, J. A., Camacho Poyato, E., \& Montesinos, P. (2017). Multiplatform application for precision irrigation scheduling in strawberries. Agricultural Water Management, 183, 194-201. https://doi.org/10.1016/j.agwat.2016.07.017

Maddock, A., Samuel Young, R., \& Reig, P. (2015). Ranking the World's Most Water-Stressed Countries in 2040. Retrieved 5 July 2018, from http://www.wri.org/blog/2015/08/ranking-world's-most-water-stressed-countries-2040

Mérida García, A., Fernández García, I., Camacho Poyato, E., Montesinos Barrios, P., \& Rodríguez Díaz, J. A. (2018). Coupling irrigation scheduling with solar energy production in a smart irrigation management system. Journal of Cleaner Production, 175, 670-682. https://doi.org/10.1016/j.jclepro.2017.12.093

Moriana, A., Orgaz, F., Pastor, M., \& Fereres, E. (2003). Yield responses of a mature olive orchard to water deficits. Journal of the American Society for Horticultural Science, 128(3), 425-431.

Orgaz, F., \& Fereres, E. (2001). El Riego. In Junta de Andalucía \& Ediciones Mundiprensa (Eds.), El Cultivo del Olivo (6ª pp. 285-306). Madrid.

Orgaz, F., Fereres, E., \& Testi, L. (2017). El Riego. In El Cultivo del Olivo (7ª pp. 461-490).

Padilla-Díaz, C. M., Rodriguez-Dominguez, C. M., Hernandez-Santana, V., Perez-Martin, A., \& Fernández, J. E. (2016). Scheduling regulated deficit irrigation in a hedgerow olive orchard from leaf turgor pressure related measurements. Agricultural Water Management, 164, 28-37. https://doi.org/10.1016/j.agwat.2015.08.002

Pagán, F. J., Ferrández-Villena, M., Fernández-Pacheco, D. G., Rosillo, J. J., \& Molina-Martínez, J. M. (2015). Optifer: An application to optimize fertiliser costs in fertigation. Agricultural Water Management, 151, $19-29$. https://doi.org/10.1016/j.agwat.2014.11.007

Palese, A. M., Celano, G., Masi, S., Xiloyannis, C., Colturali, S., Lucano, A., ... Viale, B. (2006). Treated municipal wastewater for irrigation of olive trees: effects on yield and oil quality.

Parra, M. A. (2017). Suelo. In El Cultivo del Olivo (pp. 251-287).

Pérez-Castro, A., Sánchez-Molina, J. A., Castilla, M., Sánchez-Moreno, J., Moreno-Úbeda, J. C., \& Magán, J. J. (2017). cFertigUAL: A fertigation management app for greenhouse vegetable crops. Agricultural Water Management, 183, 186193. https://doi.org/10.1016/j.agwat.2016.09.013

Petousi, I., Fountoulakis, M. S., Saru, M. L., Nikolaidis, N., Fletcher, L., Stentiford, E. I., \& Manios, T. (2015). Effects of reclaimed wastewater irrigation on olive (Olea europaea L. cv. 'Koroneiki') trees. Agricultural Water Management, 160, 33-40. https://doi.org/10.1016/j.agwat.2015.06.003

Pratap, R. (2017). Getting started with Matlab. A quick introduction for scientist and engineers. (Oxford University Press, Ed.) (7th ed.).

Rallo, L., \& Cuevas, J. (2017). Fructificación y producción. In El Cultivo del Olivo (7a, pp. 145-186). 
Rossman, L. A. (2000). EPANET 2 Users Manual. US Environmental Protection Agency (EPA), USA. https://doi.org/10.1177/0306312708089715

Segal, E., Dag, A., Ben-Gal, A., Zipori, I., Erel, R., Suryano, S., \& Yermiyahu, U. (2011). Olive orchard irrigation with reclaimed wastewater: Agronomic and environmental considerations. Agriculture, Ecosystems and Environment, 140(3-4), 454-461. https://doi.org/10.1016/j.agee.2011.01.009 